\title{
Anaplastic Oligodendroglioma
}

\author{
Jaishri Blakeley, MD and Stuart Grossman, MD
}

\section{Opinion statement}

Although uncommon, anaplastic oligodendrogliomas (AODs) are important to recognize, as they have unique molecular, histologic, and clinical features. Patients with new seizures or new focal neurologic deficits should be referred for brain MRI with contrast. If the MRI suggests a malignant glioma, maximal feasible tumor resection is advised for accurate diagnosis and for relief of tumor-related neurologic symptoms. Radiation therapy (XRT) is the most commonly prescribed postsurgical therapy for patients with AODs. The role and timing of adjuvant chemotherapy are less clear. Tumor responses to PCV (the combination of procarbazine, lomustine, and vincristine) and to temozolomide have been documented in patients with AODs. However, two prospective phase 3 trials in patients with newly diagnosed AOD have shown no difference in overall survival when PCV is added to XRT. Ongoing trials investigating the benefit of temozolomide plus XRT in patients with newly diagnosed AOD will inform about the value of this common practice.

The recognition that $1 \mathrm{p} 19 \mathrm{q}$ codeletion is a marker of oligodendroglial differentiation and the subsequent prospective confirmation of this marker's importance in predicting better prognosis have been critical discoveries. Tumors with intermediate oligodendroglial features or mixed astrocytic features should be referred for $1 \mathrm{p} 19 \mathrm{q}$ assessment. Identification of $1 \mathrm{p} 19 \mathrm{q}$ status is also required in clinical trials for patients with $\mathrm{AOD}$, given the association of $1 \mathrm{p} 19 \mathrm{q}$ codeletion with improved response to therapies and overall prognosis. There are not yet sufficient data to guide individual treatment planning based on 1p19q status, but several planned and ongoing trials will address this issue. Unfortunately, AOD remains a terminal brain cancer even with maximal therapies. As more therapeutic options become available and the full significance of molecular markers is understood, $1 \mathrm{p} 19 \mathrm{q}$ and other markers are expected to help guide optimal antitumor therapies, and it is hoped that survival and function will improve for all patients with AOD.

\section{Introduction}

Malignant gliomas are composed of neoplastic astrocytes and oligodendrocytes. Based on morphologic features, the World Health Organization (WHO) has categorized them into astrocytoma, oligodendroglioma (OD), and mixed oligoastrocytoma (MOA) [1]. Within each classification, the tumors are ordered to reflect low grade (WHO grade II) or high grade (WHO grade III or IV) based on histologic features (including high cellularity, the

Corresponding author. Jaishri Blakeley, MD, Brain Cancer Program, Johns Hopkins University, Cancer Research Building 2, 1550 Orleans, Suite 1M16, Baltimore, MD 21231, USA. jblakel3@jhmi.edu.

Disclosures

No potential conflicts of interest relevant to this article were reported. 
presence of mitotic figures, or irregular vasculature) and their predilection for aggressive behavior (Table 1). Although this classification scheme is clinically useful in most cases, the distinction of the various subtypes and grades is subjective, with high interrater variability. Moreover, it remains unclear whether various phenotypes of glioma reflect distinct diseases or heterogeneous expressions of neoplasia derived from a shared glial lineage [1, Class III;

2, Class II]. Recent discoveries in the molecular features of gliomas have raised the possibility of more accurate classification of tumors based on molecular diagnostics [1, Class III]. The identification of specific genetic markers of anaplastic oligodendroglioma (AOD) that correlate with response to therapy has been the most important of these discoveries to date and has resulted in dramatically increased interest in AOD in the past 15 years [3, Class II; 4, Class III].

Oligodendroglial tumors are the least common of the gliomas, accounting for $3 \%$ to $20 \%$ of all glial tumors [4, Class III]. Of these, roughly $70 \%$ are low-grade OD and 30\% are AOD [5, Class II]. About 20 years ago, it was recognized that tumors with oligodendroglial components have improved responses to therapy and better prognosis than other malignant gliomas [6, 7, Class III]. Since that time, the reported incidence of oligodendroglial tumors has risen steadily, with a corresponding decrease in the incidence of astrocytic tumors [8, Class II]. This change is thought to be due in part to less stringent histologic criteria for oligodendroglial tumors in the most recent WHO classification scheme as well as to a bias in favor of presenting the most favorable diagnosis to patients whenever feasible [8, Class II; 9, Class III]. More recently, however, the validation of genetic markers for identifying oligodendroglial tumors has curbed the frequency of diagnosis of AOD.

\section{HISTOLOGY}

Oligodendroglial tumors have unique histologic features. Low-grade OD (WHO grade II) has a pathognomonic histology that includes monotonous, regular, rounded, welldemarcated cells with abundant clear cytoplasm and a perinuclear halo, lending them an appearance classically referred to as "fried eggs." These cells are often seen in conjunction with a background of a fine mesh of vasculature that is thought to resemble "chicken wire" [10••, Class III]. AODs (WHO grade III) often arise from low-grade OD and hence share many of these features. However, AODs also have markers of malignancy, including mitotic figures, high cellular density, irregular cells, and endothelial hyperplasia and proliferation. There are no firm diagnostic criteria to differentiate AOD from low-grade OD beyond the recognition of the predominance of these features of anaplasia. Hence, there may be a continuum between these classifications that can complicate grading $[4,10 \bullet$, Class III].

MOAs are gliomas that have histologic features of both oligodendroglial and astrocytic tumors. MOAs may include tumors with isolated areas of predominantly astrocytes or oligodendrocytes, or tumors with heterogeneity of cells throughout the sample. There are no strict criteria for the ratio of astrocytic and oligodendroglial features that is required to label a sample MOA rather than pure oligodendroglial or astrocytic tumor. For these reasons, MOAs are among the most difficult tumors to assess histologically [10•, Class III]. Although they have been recognized as their own subcategory in the WHO classification, it is not clear whether they represent a unique histologic diagnosis or are an extension of the 
continuum of tumors derived from glial progenitor cells. The latter hypothesis is favored based on their mixed histologic features, their primarily astrocytic molecular markers, and clinical behavior that is intermediate between AOD and anaplastic astrocytoma (AA) [4, Class III].

Limitations of tumor classification based on histology include interobserver variability for tumors such as MOA and clinical bias potentially influencing the distinction of oligodendroglial tumors from astrocytic tumors. However, histology remains the most validated and practical diagnostic approach; it is accurate in predicting the clinical course for most gliomas. Recent advances in molecular testing may further enhance diagnostic accuracy in challenging glioma cases and in identification of genetic subpopulations of gliomas that are likely to respond to specific therapies.

\section{GENETICS}

The recognition that codeletion of the short arm of chromosome 1 (1p) and the long arm of chromosome 19 (19q) is a diagnostic marker for oligodendroglial tumors that is associated with improved clinical outcome was a pivotal discovery [11, Class II]. It has subsequently been validated in two prospective trials as a prognostic marker for patients with AOD and anaplastic MOA [12••, 13••, Class I]. 1p19q deletion is the most common chromosomal lesion in oligodendroglial tumors; it may be seen in as many as $60 \%$ to $90 \%$ of low-grade OD and $50 \%$ to $70 \%$ of AOD [4, 14, Class III]. It is also the earliest genetic change in these tumors and persists at the time of tumor progression or recurrence [11, Class II; 14, Class III; 15, Class II]. These findings suggest that the codeletion may be a marker of a genetically clonal cancer cell population with unique clinical features. In support of this hypothesis, 1p19q codeletion has been reliably associated with classic oligodendroglial histologic features; specific MRI findings; tumor locations, growth patterns, and response to therapies; and ultimately, survival $[4,10 \bullet$, Class III; 11 , Class II; $12 \bullet \bullet, 13 \bullet \bullet$, Class I; 15-17, 18•, Class II] (Table 2).

These findings have led to increasing clinical application of genetic profiling of gliomas. Techniques such as loss of heterozygosity ( $\mathrm{LOH}$ ) analysis using microsatellite markers, fluorescence in situ hybridization (FISH) analysis, and comparative genome hybridization are feasible in routine clinical practice and are reasonably reliable [10••, Class III]. However, there is not yet wide agreement about the value of routine testing of $1 \mathrm{p} 19 \mathrm{q}$ status [19, Class II]. There are not yet sufficient prospective data to permit treatment planning based on 1p19q status alone. However, 1p19q testing can be invaluable for some patients. It is particularly useful to help define the underlying tumor type in those with ambiguous glial histologic features. Hence, $1 \mathrm{p} 19 \mathrm{q}$ testing is recommended by most experts to confirm diagnosis in patients with oligodendroglial features $[1,4,10 \bullet \bullet$, Class III]. In the setting of clinical trials, patients with $1 \mathrm{p} 19 \mathrm{q}$ codeletion should be considered separately from those with intact alleles, given the demonstrated difference in treatment response and prognosis between these groups [16, Class II]. Ongoing trials that stratify patients based on 1p19q status are expected to provide needed information about the value of genetic profiling in clinical practice. As data regarding the clinical significance of $1 \mathrm{p} 19 \mathrm{q}$ deletion continue to 
mature, it is likely that $1 \mathrm{p} 19 \mathrm{q}$ testing will be included in the diagnosis of oligodendroglial tumors.

The significance of isolated $1 \mathrm{p}$ or $19 \mathrm{q}$ loss remains unclear. Some data suggest that isolated $1 \mathrm{p}$ deletion is associated with improved treatment response and prognosis, but not to the same extent as combined deletion with 19q [3, Class II]. There is no apparent prognostic benefit with isolated 19q deletion [3, Class II]. New evidence shows that a whole-arm (centrosomic and pericentrosomic) translocation of $1 \mathrm{p}$ and $19 \mathrm{q}$ alleles may be a critical genetic event that defines oligodendroglial tumors [20•, Class II]. As mentioned, $1 \mathrm{p} 19 \mathrm{q}$ status appears to be preserved across the life of the tumor and is independent of grade or recurrence $[15,17$, Class II]. Collectively, these data suggest that codeletion of $1 \mathrm{p} 19 \mathrm{q}$ may be the causal event in the genesis of a subtype of oligo dendroglial tumors and that combined loss of $1 \mathrm{p} 19 \mathrm{q}$ (possibly via translocation) defines a specific tumor type associated with a more favorable prognosis.

Additional molecular changes in AOD support the hypothesis that tumors with 1p19q codeletion are distinct from other glial tumors. Genetic abnormalities, including the deletion of the cyclin-dependent kinase inhibitor gene CDKN2C (chromosome 9p), TP53 mutation (chromosome 17p), and epidermal growth factor receptor $(E G F R)$ amplification have been identified in some AOD [3, 11, Class II; 14, Class III]. Compared with 1p19q LOH, these mutations are all associated with a worse prognosis. In fact, there is an inverse relationship between 1p19q codeletion and the presence of TP53, 10q, and PTEN mutations [3, Class II]. Clinical outcome similarly is most favorable in the setting of $1 \mathrm{p} 19 \mathrm{q} \mathrm{LOH}$ and least favorable with TP53, 10q, or PTEN mutations [3, Class II; 4, Class III]. Because 1p19q LOH is not fully predictive of treatment response or prognosis, efforts are ongoing to identify and validate additional markers that may increase diagnostic accuracy. To date, however, $1 \mathrm{p} 19 \mathrm{q}$ analysis is the only molecular test that has been prospectively validated in AOD.

Anaplastic MOA may share some molecular features with AOD. However, most MOAs do not have $1 \mathrm{p} 19 \mathrm{q}$ co deletion, but rather express markers more commonly seen in pure astrocytic tumors including TP53, 10q, and PTEN mutations [15, Class II]. Their clinical course reflects the significance of these markers, as fewer cases of anaplastic MOA are responsive to chemotherapy, and overall survival (OS) for patients with anaplastic MOA without 1p19q is more similar to that seen with AA than with AOD.

\section{CLINICAL PRESENTATION}

AOD tumors occur most commonly in adults. The peak age of onset is 35 to 44 years, with low-grade OD presenting in younger patients and AOD presenting in older patients [5, Class II]. Oligodendroglial tumors are very rare in children, accounting for only $2 \%$ to $4 \%$ of primary pediatric brain tumors; the great majority of these are low-grade [5, Class II]. In both children and adults, there is a slight predilection for OD tumors in males, with a male to female ratio of roughly 1.4:1.

AOD occur in white matter and the cortex. Their location is most commonly supratentorial, particularly in the frontal lobes, but they can present throughout the central nervous system, including the brainstem and spinal cord. When oligodendroglial tumors are found 
infratentorially or in the deep nuclei, they are generally thought to represent a more aggressive phenotype [4, Class III]. AOD almost always present as single lesions. There are case reports of multifocal AOD, leptomeningeal spread of AOD, and extraneural metastases from AOD. However, these cases are rare and may simply reflect the natural history of a glioma associated with prolonged survival [4, 21, Class III].

The clinical presentation largely depends on the tumor's location and grade. Seizures are the most common symptom at presentation for all OD (50\%-90\% of patients) [4, 22, Class III]. Seizures are particularly common with low-grade OD, which grow slowly and cause irritation before symptoms due to mass effect. AODs may also present with seizures but are more likely to present with acute to subacute onset of neurologic symptoms such as headache, focal weakness, vision changes, or cognitive deficits due to mass effect from the tumor.

On both CT and MRI, most AODs have enhancement after administration of exogenous contrast. This represents an abnormal blood-brain barrier within the tumor and possibly tumor angiogenesis. MRI is the preferred modality for assessing AOD because of its superior ability to assess the neuroanatomic location of tumor and the extent of signal abnormality. T2-weighted MRI often shows a mixed-intensity central core with diffuse surrounding signal hyperintensity [18•, Class II]. The T1-weighted sequence without gadolinium often shows hypointensity, but hyperintensity may be seen if calcification is present. CT is often needed to confirm calcification. The presence of calcifications on neuroimaging is likely to be a marker of long-standing or chronic disease rather than a specific biologic effect. The presence of calcification has been correlated with 1p19q LOH [18•, 23, Class II]. AODs may also be associated with spontaneous intratumoral hemorrhage, and some have suggested that this is more likely in AOD than in other gliomas [24, 25, Class III]. It is possible that hemorrhage is related to the specific microstructure of OD vasculature, but this connection has not been confirmed [25, Class III]. Finally, unlike other malignant gliomas, oligodendroglial tumors are often noted on MRI to have sharp demarcations between normal brain and tumor core. Recently, this imaging feature was correlated with 1p19q status: patients with 1p19q LOH were more likely to have a diffuse tumor border on MRI, whereas patients with intact alleles had the appearance of a sharp border [18•, 23, Class II].

Functional MRI techniques have recently been applied to AOD. There appears to be increased thallium-201 uptake on MR single-photon emission computed tomography (SPECT) in AOD patients with $\mathrm{LOH}$ at 1p19q regardless of grade [17, Class II]. However, functional MRI techniques have not yet been validated to distinguish subtypes of oligodendroglial tumors, and the clinical relevance of this observation is not yet clear.

\section{Treatment}

\section{Surgery}

- Maximal feasible tumor resection is first-line therapy for AOD. Surgery provides tissue to confirm the diagnosis and to perform additional histologic and genetic testing to determine the glioma subtype. In patients who present with neurologic 
symptoms due to mass effect, debulking may provide significant improvement in neurologic function. For patients with low-grade OD, it may also offer relief of tumor-related seizures and may potentially prolong the time to recurrence or progression [26, 27, Class III]. Hence, surgical resection is indicated as first-line therapy for oligodendroglial tumors, for both diagnosis and improvement of clinical symptoms.

- The extent of surgical resection required is highly controversial. It is widely believed that large-volume resection is optimal, as it provides the greatest volume of tumor for pathologic testing (avoiding the confounding factor of sampling error), reduces mass effect contributing to neurologic dysfunction, and provides tumor cytoreduction that potentially may improve the efficacy of adjuvant therapies. However, AODs are widely infiltrative tumors that currently cannot be cured with surgery, and they often involve critical areas of the brain. Postoperative neurologic complications may reduce performance status. As performance status is a wellrecognized, powerful prognostic factor and an important determinant of quality of life (QOL), every effort must be made to ensure that patients are as neurologically intact as possible.

- Several reports suggest that radical tumor resection (> 90\% of contrast-enhancing volume) results in improved progression-free survival (PFS) and OS in both adults and children across all malignant gliomas [28, 29, Class III]. However, all of these investigations are uncontrolled (and often retrospective), and it is possible that tumor location, patient features such as age and comorbid disease, and basic tumor biology account for the observed differences in survival after radical versus partial resection or biopsy alone. Moreover, the data regarding the relationship between degree of resection and OS do not appear to differ for AOD versus other glioma subtypes. Studies in which patients with AOD are reported independently have had mixed results, with some suggesting no difference in survival after complete versus partial resection or biopsy and others suggesting a survival benefit with maximal resection [30, Class III]. Overall, it is recommended that patients with AOD undergo resection of the largest volume of tumor possible while minimizing the risks of postoperative neurologic deficit.

\section{Radiation therapy}

- Postoperative XRT, the standard of care for malignant gliomas such as glioblastoma multiforme (GBM) and AA, is similarly widely considered to be the

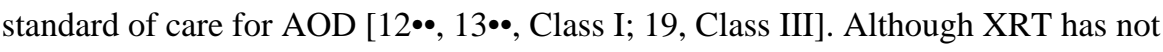
been investigated prospectively specifically for AOD, it has been shown in randomized, controlled trials to be an effective therapy for high-grade gliomas of all subtypes. Moreover, several retrospective studies have suggested the efficacy of $\mathrm{XRT}$ for AOD, and two recent randomized, prospective trials have confirmed its

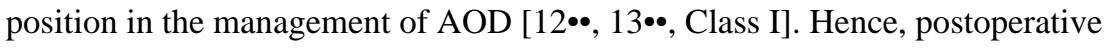
XRT to a total dose of roughly 60 Gy over 30 fractions is recommended for patients with AOD. 
- Recently, some specialists have suggested that XRT could potentially be omitted for patients with AOD and 1p19q codeletion [3, Class III; 31, Class II]. A recent survey showed that up to $34 \%$ of neuro-oncologists recommended delaying XRT in such patients [19, Class II]. This practice is based on the observed responsiveness of these tumors to chemotherapy and on the concern about long-term neurocognitive effects that may be associated with XRT. However, there are currently no prospective data to support delaying or omitting XRT for patients with AOD. Given that AODs are infiltrative malignant gliomas that are invariably fatal, it seems prudent to offer standard therapy for malignant gliomas until additional evidence is available. Clinical trials to address the efficacy of delayed XRT are planned, and patients with $1 \mathrm{p} 19 \mathrm{q} \mathrm{LOH}$ should be considered for these trials to better address this important question.

- The role of XRT in low-grade OD is controversial. In a randomized, controlled trial of early versus delayed XRT for all low-grade gliomas, there was no difference in OS if XRT was delayed until the time of progression (defined as changes on CT) [32, Class I]. PFS was improved, but it was not clear from study results whether this prolonged PFS was associated with better patient performance. These data and prior reports on the response to XRT of patients with low-grade OD support an approach in which patients with low-grade OD who have undergone complete resection and have no additional tumor-related neurologic deficits have XRT deferred until the time of clinical progression [32, Class I; 33-35, Class III]. In contrast, patients with large volumes of residual tumor or neurologic deficits are likely to benefit from early postoperative XRT [34, 36, Class III].

\section{Pharmacologic treatment}

- AOD is unique among the malignant gliomas in being responsive to a range of chemotherapies. This response was first recognized by Cairncross and Macdonald [6, Class III], who reported eight patients with AOD that showed radiographic and clinical response to chemotherapy at the time of first recurrence after XRT. Initial studies suggested that AOD may be particularly sensitive to combined chemotherapy with procarbazine, lomustine, and vincristine (PCV), but a growing body of literature suggests that AODs are responsive to a variety of therapies (Table 3).

\section{Procarbazine, lomustine, and vincristine (PCV)}

- Several uncontrolled, phase 2 studies have confirmed the initial observation that AOD are more responsive to PCV than other gliomas and that AOD respond to PCV both at the time of diagnosis and at first recurrence after XRT [7, 37-39, Class II]. However, uncertainty remained about the optimal timing of PCV for these tumors. Two recent randomized, phase 3 trials investigated the benefit of PCV therapy in newly diagnosed AOD. The Radiation Therapy Oncology Group (RTOG) investigated the benefit of PCV prior to XRT (RTOG 9402) [13••, Class I]. The European Organisation for Research and Treatment of Cancer (EORTC) investigated PCV given after XRT (EORTC 26951) [12••, Class I] (Table 3). The 
two trials showed very similar results. In both trials, PCV added to XRT resulted in improved PFS, but there was no significant difference in OS. In the EORTC trial, $79 \%$ of patients randomized to XRT alone received chemotherapy at the time of progression [12••, Class I]. Hence, the studies showed that there is no apparent difference in OS between up-front PCV versus PCV at the time of recurrence in patients with AOD treated with XRT. In both studies, the improvement in PFS was associated with significant toxicity attributed to the PCV: $20 \%$ to $33 \%$ of the patients could not complete the prescribed therapy because of chemotherapyrelated toxicity. A follow-up study assessed patient-reported QOL during the EORTC study and showed no difference in QOL between the XRT-only group and the XRT-plus-PCV group [40, Class II]. Hence, although PFS was prolonged with the addition of PCV, there was no concomitant improvement in QOL when adjuvant chemotherapy was given. In addition, the PCV toxicity contributed to decreased QOL for a period of time. As a result of these two decades of careful research, PCV therapy is not currently recommended as first-line therapy for AOD.

- Importantly, both RTOG 9402 and EORTC 26951 evaluated the 1p19q status prospectively and confirmed that $1 \mathrm{p} 19 \mathrm{q}$ codeletion results in better OS in patients

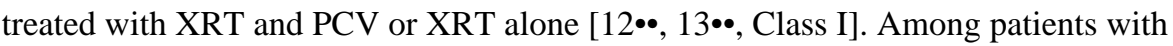
$1 \mathrm{p} 19 \mathrm{q}$ codeletion, there was no difference in OS when PCV was added before or after XRT [12••, Class I]. These data support the hypothesis that 1p19q codeletion predicts better overall prognosis in patients with AOD given standard therapeutic modalities (surgery, XRT, PCV), but not necessarily better response to any one specific therapy.

\section{Temozolomide}

- Temozolomide (TMZ) is an oral alkylating therapy that is better tolerated than PCV and has been demonstrated to cross the blood-brain barrier. It has been shown to improve OS when given with XRT and as an adjuvant after XRT versus XRT alone in patients with newly diagnosed GBM [41, Class I].

- $\quad$ Recent trials have demonstrated that AOD and anaplastic MOA are responsive to TMZ [42-44, Class II]. This was first confirmed by Yung et al. [45, Class II] in a mixture of patients with progressive anaplastic MOA, AOD, or OD. The PFS was 5.8 months for patients with anaplastic MOA. Of note, all patients had prior XRT and $69 \%$ had prior nitrosourea-based therapies. Chinot et al. [42, Class II] showed that patients with AOD or anaplastic MOA previously treated with XRT and subsequent PCV had an objective rate of response to TMZ of 44\%; median OS was 10 months. Subsequently, van den Bent et al. [43, Class II] confirmed response to TMZ in patients with AOD or anaplastic MOA previously treated with PCV, but the response was somewhat less robust, at only $25 \%$. Methodologic differences and possibly prognostic variables such as $1 \mathrm{p} 19 \mathrm{q}$ status explain these differences. Importantly, TMZ was well tolerated in these heavily treated populations; most patients completed the prescribed doses.

- $\quad$ TMZ has also been investigated as first-line chemotherapy for oligodendroglial tumors progressive after XRT. van den Bent et al. [46, Class II] showed that TMZ 
at the time of first recurrence after XRT resulted in a 53\% response rate and a median 12-month PFS of 40\%. Similarly, Brandes et al. [44, Class II] reported that TMZ had a response rate of $46 \%$ in patients with AOD or anaplastic MOA progressive after surgery and XRT. In this study, 1p19q status and O6methylguanine DNA methyltransferase (MGMT) status were recorded. Methylation of MGMT renders it inactive, making the cell less able to repair alkylating injuries (eg, from TMZ or PCV) and resulting in increased cell death. It has been hypothesized that one of the reasons AODs are more responsive to PCV and TMZ than other gliomas is enhanced MGMT methylation. Brandes et al. [44, Class II] found that $1 \mathrm{p} 19 \mathrm{q} \mathrm{LOH}$ was correlated with response to TMZ and OS similar to those in other reports. Further, MGMT methylation was associated with $1 \mathrm{p} 19 \mathrm{q}$ codeletion. However, MGMT methylation was not directly associated with improved response or enhanced survival.

- $\quad$ TMZ has been applied up front with XRT in patients with newly diagnosed AOD because of three observations: 1) AOD responds to TMZ at the time of both first and second recurrence; 2) TMZ plus XRT is effective in newly diagnosed GBM; and 3) overall, TMZ is well tolerated [19, Class II]. However, there are currently no prospective data to support this practice. In the RTOG 0131 trial, patients with AOD or anaplastic MOA are treated with neoadjuvant TMZ for 6 months, followed by TMZ and concurrent XRT [47, Class II]. The interim report suggests that combination therapy with TMZ and XRT is well tolerated by these patients. Assessment of $1 \mathrm{p} 19 \mathrm{q}$ status revealed that PFS was improved in patients with $1 \mathrm{p} 19 \mathrm{q}$ codeletion versus those with intact $1 \mathrm{p} 19 \mathrm{q}$ alleles who received this regimen [47, Class II]. OS data are pending. Final results from this study and ongoing clinical trials combining TMZ and XRT at the time of diagnosis will address the safety and efficacy of this approach for patients with AOD.

\section{Chemotherapy and recurrent disease}

- At the time of recurrence, AOD tumors are sensitive to multiple agents, with documented response rates of 53\% to TMZ and 50\% to PCV [9, Class III]. Since TMZ is better tolerated than PCV, it has become first-line for chemotherapy-naive patients with recurrent or progressive AOD. Patients with progressive AOD who have previously been treated with TMZ are less responsive to PCV as salvage therapy, with response rates of only $10 \%$ to $20 \%$ [48 , Class II]. Similarly, patients with AOD that progressed on PCV had a response rate to TMZ of 25\% [43, Class II]. Several other agents have been tried in recurrent AOD with variable results. Reports in the literature list numerous agents that have resulted in at least transient tumor response, including carmustine, carboplatin, etoposide, cisplatin, melphalan, thiotepa, and paclitaxel [49, Class III]. However, all these reports have involved limited, retrospective series, and benefit has not been proven. Hence, patients with recurrent AOD after standard therapies may be good candidates for research protocols. 


\title{
Chemotherapy with deferred radiation therapy
}

- The observation that oligodendroglial tumors with $1 \mathrm{p} 19 \mathrm{q}$ codeletion are responsive to chemotherapy has led to the suggestion that chemotherapy can be used as firstline therapy in patients with newly diagnosed AOD with 1p19q deletion, deferring XRT for progression [3, Class II; 31, Class III]. This suggestion is based in part on concern that XRT has a risk of long-term cognitive injury that may be particularly salient in patients expected to have long survival. However, no survival data are available to support this hypothesis, and the true impact of modern XRT approaches on cognition is not clear.

- In the ongoing RTOG 0131 trial, early reports show a 33\% rate of complete or partial response to TMZ prior to XRT; only $10 \%$ of patients with newly diagnosed AOD or anaplastic MOA progressed while on TMZ monotherapy [47, Class II]. Again, response was correlated with 1p19q status. In this trial, however, all patients go on to receive XRT; hence the duration of response and OS with up-front chemotherapy and deferred XRT will not be assessed. Other trials using TMZ monotherapy in patients with newly diagnosed AOD or anaplastic MOA are ongoing, using various TMZ dosing schedules. The final results of the RTOG 0131 trial and complementary trials with up-front therapy with or without concurrent XRT will help to better define the optimal combination of XRT and chemotherapy for patients with newly diagnosed oligodendroglioma.

- In the interim, it is most prudent to offer standard therapy for malignant gliomas to all patients with AOD or anaplastic MOA. Given that the optimal therapy for AOD both at the time of diagnosis and at recurrence remains unclear, patients with AOD at all stages of disease are excellent candidates for clinical investigations and should be encouraged to explore participation in promising trials.

\section{Pharmacologic treatment \\ Procarbazine}

\begin{aligned} & \hline $\begin{array}{r}\text { Standard dosage } \\
\text { Contraindications }\end{array} \begin{array}{l}60 \mathrm{mg} / \mathrm{m}^{2} \text { daily on days 8-21. } \\
\text { Hypersensitivity to drug or components, myelosuppression. }\end{array} \\
&$ Main drug interactions $\begin{array}{l}\text { Disulfiram-like interaction with alcohol. Tricyclic antidepressants and sympathomimetics } \\
\text { may induce hypertensive crisis. Rare cases of encephalopathy. }\end{array} \\
&$ Main side effects $\begin{array}{l}\text { Encephalopathy, nausea, vomiting, myelosuppression, photosensitivity. } \\
\text { Special points }\end{array} \\
& \begin{array}{l}\text { Weak monoamine oxidase inhibitor requiring patients to avoid foods that are tyramine-rich. } \\
\text { Best taken on an empty stomach at bedtime. Dosed in 50-mg tablets; a patient's individual } \\
\text { dose should be rounded to the nearest 50 mg based on dry weight. } \\
\text { Cost }\end{array}$\begin{tabular}{l} 
\$24 per pill, roughly \$1000 per cycle. \\
\hline
\end{tabular} \\
& \hline\end{aligned}

\section{Lomustine (CCNU)}

\author{
Standard dosage $110 \mathrm{mg} / \mathrm{m}^{2}$ on day 1 ; repeat every 8 weeks. \\ Contraindications Hypersensitivity to nitrosoureas, inadequate bone marrow function, history of pulmonary \\ fibrosis.
}




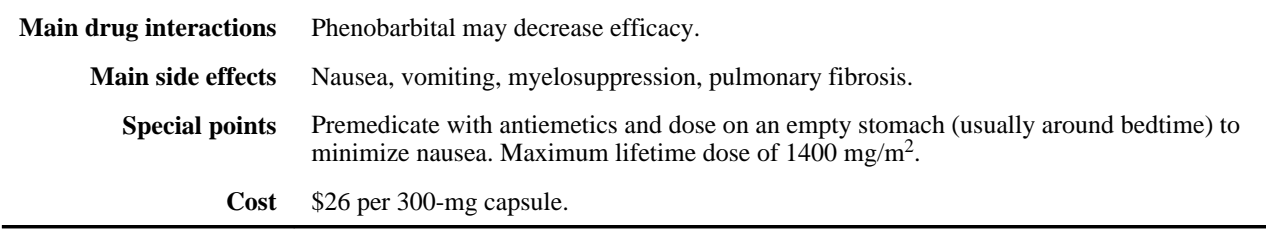

\section{Vincristine}

\begin{tabular}{|c|c|}
\hline Standard dosage & $1.4 \mathrm{mg} / \mathrm{m}^{2}$ (intravenous) on days 8 and 29 . \\
\hline Contraindications & $\begin{array}{l}\text { Hypersensitivity to the drug or components, intrathecal administration, history of familial or } \\
\text { other advanced peripheral neuropathy. }\end{array}$ \\
\hline Main drug interactions & Cytochrome P450 inhibitors may increase vincristine serum concentrations. \\
\hline Main side effects & Neuropathy (peripheral or autonomic) is the most common, dose-limiting toxicity. \\
\hline Special points & $\begin{array}{l}\text { There is debate about the efficacy of vincristine for many adult primary tumors, particularly } \\
\text { because preclinical models have shown that it has very little access to the central nervous } \\
\text { system. It is difficult to tolerate and requires special precautions for administration because } \\
\text { of its vesicant activity. Hence, in some centers procarbazine and lomustine are offered } \\
\text { without vincristine. }\end{array}$ \\
\hline Cost & About $\$ 40$ per infusion. \\
\hline
\end{tabular}

\section{PCV combination therapy}

Standard dosage Given every 6-8 weeks in the following order: CCNU $\left(110 \mathrm{mg} / \mathrm{m}^{2}\right)$ on day 1 ; intravenous vincristine $\left(1.4 \mathrm{mg} / \mathrm{m}^{2}\right)$ on days 8 and 29 ; oral procarbazine $\left(60 \mathrm{mg} / \mathrm{m}^{2}\right)$ daily on days $8-21$.

\section{Temozolomide}

\begin{aligned} & \hline Standard dosage $\begin{array}{l}75 \mathrm{mg} / \mathrm{m}^{2} \text { daily for about } 42 \text { consecutive days when given with XRT. The adjuvant dosage } \\ \text { is } 150-200 \mathrm{mg} / \mathrm{m}^{2} \text { for } 5 \text { of every } 28 \text { days. }\end{array} \\ &$ Main drug interactions $\begin{array}{l}\text { Hypersensitivity to TMZ or dacarbazine; pregnancy, nursing. } \\ \text { Administration of valproic acid decreases oral clearance of TMZ by about 5\%; the clinical } \\ \text { implication of this effect is not known. }\end{array} \\ &$ Main side effects $\begin{array}{l}\text { Nausea, vomiting, myelosuppression (thrombocytopenia). } \\ \text { Special points }\end{array} \\ & \begin{array}{l}\text { Available in 5-mg, 20-mg, 100-mg, 140-mg, 180-mg and 250-mg doses. Doses rounded up } \\ \text { to the nearest } 10 \mathrm{mg} \text {. During XRT, should be taken } 1 \text { hour before XRT. In the adjuvant } \\ \text { phase, best taken at bedtime on an empty stomach. } \\ \text { About } \$ 400 \text { per 250-mg capsule (\$13,000 for use concurrent with XRT therapy, \$2000/mo } \\ \text { for adjuvant therapy). }\end{array} \\ &$\hline\end{aligned}

\section{References and Recommended Reading}

Papers of particular interest, published recently, have been highlighted as:

- Of importance

•• Of major importance

1. Louis DN, Holland EC, Cairncross JG. Glioma classification: a molecular reappraisal. Am J Pathol. 2001; 159:779-786. [PubMed: 11549567] 
2. Maintz D, Fiedler K, Koopmann J, et al. Molecular genetic evidence for subtypes of oligoastrocytomas. J Neuropathol Exp Neurol. 1997; 56:1098-1104. [PubMed: 9329453]

3. Ino Y, Zlatescu MC, Sasaki H, et al. Long survival and therapeutic responses in patients with histologically disparate high-grade gliomas demonstrating chromosome 1p loss. J Neurosurg. 2000; 92:983-990. [PubMed: 10839259]

4. van den Bent MJ. Anaplastic oligodendroglioma and oligoastrocytoma. Neurol Clin. 2007; 25:1089-1109. ix-x. [PubMed: 17964027]

5. CBTRUS. Statistical report: Primary brain tumors in the United States, 1998-2002. Published by the Central Brain Tumor Registry of the United States 2005. Available at http://www.cbtrus.org/ reports//2005-2006/2006report.pdf.

6. Cairncross JG, Macdonald DR. Successful chemotherapy for recurrent malignant oligodendroglioma. Ann Neurol. 1988; 23:360-364. [PubMed: 3382171]

7. van den Bent MJ, Kros JM, Heimans JJ, et al. Response rate and prognostic factors of recurrent oligodendroglioma treated with procarbazine, CCNU, and vincristine chemotherapy. Dutch Neurooncology Group. Neurology. 1998; 51:1140-1145. [PubMed: 9781544]

8. McCarthy BJ, Propp JM, Davis FG, Burger PC. Time trends in oligodendroglial and astrocytic tumor incidence. Neuroepidemiology. 2008; 30:34-44. [PubMed: 18259099]

9. van den Bent M, Chinot OL, Cairncross JG. Recent developments in the molecular characterization and treatment of oligodendroglial tumors. Neuro Oncol. 2003; 5:128-138. [PubMed: 12672285]

10. Aldape K, Burger PC, Perry A. Clinicopathologic aspects of $1 \mathrm{p} / 19 \mathrm{q}$ loss and the diagnosis of oligodendroglioma. Arch Pathol Lab Med. 2007; 131:242-251. [PubMed: 17284109] Concise review of the current state of the field for pathologic diagnosis of oligodendroglioma.

11. Cairncross JG, Ueki K, Zlatescu MC, et al. Specific genetic predictors of chemotherapeutic response and survival in patients with anaplastic oligodendrogliomas. J Natl Cancer Inst. 1998; 90:1473-1479. [PubMed: 9776413]

12. van den Bent MJ, Carpentier AF, Brandes AA, et al. Adjuvant procarbazine, lomustine, and vincristine improves progression-free survival but not overall survival in newly diagnosed anaplastic oligodendrogliomas and oligoastrocytomas: a randomized European Organisation for Research and Treatment of Cancer phase III trial. J Clin Oncol. 2006; 24:2715-2722. [PubMed: 16782911] Seminal paper confirming the prognostic power of $1 \mathrm{p} 19 \mathrm{q}$ codeletion and confirming no survival benefit with adjuvant PCV for newly diagnosed AODs.

13. Cairncross G, Berkey B, Shaw E, et al. Phase III trial of chemotherapy plus radiotherapy compared with radiotherapy alone for pure and mixed anaplastic oligodendroglioma: Intergroup Radiation Therapy Oncology Group trial 9402. J Clin Oncol. 2006; 24:2707-2714. [PubMed: 16782910] Pivotal paper confirming 1p19q LOH as a marker for good prognosis for oligodendroglioma and no survival benefit with adjuvant chemotherapy for patients with newly diagnosed AOD.

14. Reifenberger G, Louis DN. Oligodendroglioma: toward molecular definitions in diagnostic neurooncology. J Neuropathol Exp Neurol. 2003; 62:111-126. [PubMed: 12578221]

15. Fallon KB, Palmer CA, Roth KA, et al. Prognostic value of 1p, 19q, 9p, 10q, and EGFR-FISH analyses in recurrent oligodendrogliomas. J Neuropathol Exp Neurol. 2004; 63:314-322. [PubMed: 15099021]

16. van den Bent MJ, Looijenga LH, Langenberg K, et al. Chromosomal anomalies in oligodendroglial tumors are correlated with clinical features. Cancer. 2003; 97:1276-1284. [PubMed: 12599236]

17. Walker C, du Plessis DG, Fildes D, et al. Correlation of molecular genetics with molecular and morphological imaging in gliomas with an oligodendroglial component. Clin Cancer Res. 2004; 10:7182-7191. [PubMed: 15534091]

18. Jenkinson MD, du Plessis DG, Smith TS, et al. Histological growth patterns and genotype in oligodendroglial tumours: correlation with MRI features. Brain. 2006; 129:1884-1891. [PubMed: 16670176] Correlation of imaging features commonly reviewed in clinical practice (tumor location, morphology on imaging, and calcification) with 1p19q status and histology. Suggests that $1 \mathrm{p} 19 \mathrm{q}$ status is a pervasive indicator of disease type across modalities.

19. Abrey LE, Louis DN, Paleologos N, et al. Survey of treatment recommendations for anaplastic oligodendroglioma. Neuro Oncol. 2007; 9:314-318. [PubMed: 17435180] 
20. Jenkins RB, Blair H, Ballman KV, et al. A t(1;19)(q10;p10) mediates the combined deletions of 1p and $19 q$ and predicts a better prognosis of patients with oligodendroglioma. Cancer Res. 2006; 66:9852-9861. [PubMed: 17047046] Evidence of matched translocation as a pathologic mechanism for $1 \mathrm{p} 19 \mathrm{q}$ loss.

21. Mork SJ, Lindegaard KF, Halvorsen TB, et al. Oligodendroglioma: incidence and biological behavior in a defined population. J Neurosurg. 1985; 63:881-889. [PubMed: 4056902]

22. Daumas-Duport C, Varlet P, Tucker ML, et al. Oligodendrogliomas. Part I: patterns of growth, histological diagnosis, clinical and imaging correlations: a study of 153 cases. J Neurooncol. 1997; 34:37-59. [PubMed: 9210052]

23. Megyesi JF, Kachur E, Lee DH, et al. Imaging correlates of molecular signatures in oligodendrogliomas. Clin Cancer Res. 2004; 10:4303-4306. [PubMed: 15240515]

24. Kondziolka D, Bernstein M, Resch L, et al. Significance of hemorrhage into brain tumors: clinicopathological study. J Neurosurg. 1987; 67:852-857. [PubMed: 3316531]

25. Liwnicz BH, Wu SZ, Tew JM Jr. The relationship between the capillary structure and hemorrhage in gliomas. J Neurosurg. 1987; 66:536-541. [PubMed: 3031239]

26. Berger MS, Deliganis AV, Dobbins J, Keles GE. The effect of extent of resection on recurrence in patients with low grade cerebral hemisphere gliomas. Cancer. 1994; 74:1784-1791. [PubMed: 8082081]

27. Chang EF, Potts MB, Keles GE, et al. Seizure characteristics and control following resection in 332 patients with low-grade gliomas. J Neurosurg. 2008; 108:227-235. [PubMed: 18240916]

28. Devaux BC, O'Fallon JR, Kelly PJ. Resection, biopsy, and survival in malignant glial neoplasms. A retrospective study of clinical parameters, therapy, and outcome. J Neurosurg. 1993; 78:767775. [PubMed: 8468607]

29. Laws ER, Parney IF, Huang W, et al. Survival following surgery and prognostic factors for recently diagnosed malignant glioma: data from the Glioma Outcomes Project. J Neurosurg. 2003; 99:467-473. [PubMed: 12959431]

30. Daumas-Duport C, Tucker ML, Kolles H, et al. Oligodendrogliomas. Part II: a new grading system based on morphological and imaging criteria. J Neurooncol. 1997; 34:61-78. [PubMed: 9210053]

31. Chinot O. Chemotherapy for the treatment of oligodendroglial tumors. Semin Oncol. 2001; 28:1318. [PubMed: 11550134]

32. van den Bent MJ, Afra D, de Witte O, et al. Long-term efficacy of early versus delayed radiotherapy for low-grade astrocytoma and oligodendroglioma in adults: the EORTC 22845 randomised trial. Lancet. 2005; 366:985-990. [PubMed: 16168780]

33. Bullard DE, Rawlings CE 3rd, Phillips B, et al. Oligodendroglioma. An analysis of the value of radiation therapy. Cancer. 1987; 60:2179-2188. [PubMed: 3440228]

34. Celli P, Nofrone I, Palma L, et al. Cerebral oligodendroglioma: prognostic factors and life history. Neurosurgery. 1994; 35:1018-1034. discussion 1034-1035. [PubMed: 7885546]

35. Fisher BJ, Leighton CC, Vujovic O, et al. Results of a policy of surveillance alone after surgical management of pediatric low grade gliomas. Int J Radiat Oncol Biol Phys. 2001; 51:704-710. [PubMed: 11597812]

36. Olson JD, Riedel E, DeAngelis LM. Long-term outcome of low-grade oligodendroglioma and mixed glioma. Neurology. 2000; 54:1442-1448. [PubMed: 10751254]

37. Cairncross G, Macdonald D, Ludwin S, et al. Chemotherapy for anaplastic oligodendroglioma. National Cancer Institute of Canada Clinical Trials Group. J Clin Oncol. 1994; 12:2013-2021. [PubMed: 7931469]

38. Soffietti R, Ruda R, Bradac GB, Schiffer D. PCV chemotherapy for recurrent oligodendrogliomas and oligoastrocytomas. Neurosurgery. 1998; 43:1066-1073. [PubMed: 9802850]

39. Buckner JC, Gesme D Jr, O'Fallon JR, et al. Phase II trial of procarbazine, lomustine, and vincristine as initial therapy for patients with low-grade oligodendroglioma or oligoastrocytoma: efficacy and associations with chromosomal abnormalities. J Clin Oncol. 2003; 21:251-255. [PubMed: 12525516]

40. Taphoorn MJ, van den Bent MJ, Mauer ME, et al. Health-related quality of life in patients treated for anaplastic oligodendroglioma with adjuvant chemotherapy: results of a European Organisation 
for Research and Treatment of Cancer randomized clinical trial. J Clin Oncol. 2007; 25:57235730. [PubMed: 18089866]

41. Stupp R, Mason WP, van den Bent MJ, et al. Radiotherapy plus concomitant and adjuvant temozolomide for glioblastoma. N Engl J Med. 2005; 352:987-996. [PubMed: 15758009]

42. Chinot OL, Honore S, Dufour H, et al. Safety and efficacy of temozolomide in patients with recurrent anaplastic oligodendrogliomas after standard radiotherapy and chemotherapy. J Clin Oncol. 2001; 19:2449-2455. [PubMed: 11331324]

43. van den Bent MJ, Chinot O, Boogerd W, et al. Second-line chemotherapy with temozolomide in recurrent oligodendroglioma after PCV (procarbazine, lomustine and vincristine) chemotherapy: EORTC Brain Tumor Group phase II study 26972. Ann Oncol. 2003; 14:599-602. [PubMed: 12649108]

44. Brandes AA, Tosoni A, Cavallo G, et al. Correlations between O6-methylguanine DNA methyltransferase promoter methylation status, $1 \mathrm{p}$ and $19 \mathrm{q}$ deletions, and response to temozolomide in anaplastic and recurrent oligodendroglioma: a prospective GICNO study. J Clin Oncol. 2006; 24:4746-4753. [PubMed: 16954518]

45. Yung WK, Prados MD, Yaya-Tur R, et al. Multicenter phase II trial of temozolomide in patients with anaplastic astrocytoma or anaplastic oligoastrocytoma at first relapse. J Clin Oncol. 1999; 17:2762-2771. [PubMed: 10561351]

46. van den Bent MJ, Taphoorn MJ, Brandes AA, et al. Phase II study of first-line chemotherapy with temozolomide in recurrent oligodendroglial tumors: the European Organization for Research and Treatment of Cancer Brain Tumor Group study 26971. J Clin Oncol. 2003; 21:2525-2528. [PubMed: 12829671]

47. Vogelbaum MA, Berkey B, Peereboom D, et al. RTOG 0131: phase II trial of pre-irradiation and concurrent temozolomide in patients with newly diagnosed anaplastic oligodendrogliomas and mixed anaplastic oligodendrogliomas: relationship between $1 \mathrm{p} / 19 \mathrm{q}$ status and progression-free survival [abstract 1517]. J Clin Oncol ASCO Annual Meeting Proceedings. 2006 Jun 20.24(Suppl)

48. Kouwenhoven MC, Kros JM, French PJ, et al. 1p/19q loss within oligodendroglioma is predictive for response to first line temozolomide but not to salvage treatment. Eur J Cancer. 2006; 42:2499 2503. [PubMed: 16914310] Analysis of first-line and second-line temozolomide EORTC trial patients for $1 \mathrm{p} 19 \mathrm{q}$ status. $1 \mathrm{p} 19 \mathrm{q}$ LOH was predictive of response to first-line, but not second-line, therapy.

49. Engelhard HH, Stelea A, Mundt A. Oligodendroglioma and anaplastic oligodendroglioma: clinical features, treatment, and prognosis. Surg Neurol. 2003; 60:443-456. [PubMed: 14572971]

50. Zlatescu MC, TehraniYazdi A, Sasaki H, et al. Tumor location and growth pattern correlate with genetic signature in oligodendroglial neoplasms. Cancer Res. 2001; 61:6713-6715. [PubMed: 11559541] 


\section{Table 1}

World Health Organization classification of tumors with oligodendroglial features

\begin{tabular}{|lc|}
\hline Class & Grade \\
\hline Oligodendroglial tumors & \\
Oligodendroglioma & II \\
Anaplastic oligodendroglioma & III \\
Mixed gliomas & \\
Oligoastrocytoma & II \\
Anaplastic oligoastrocytoma & III \\
\hline
\end{tabular}




\section{Table 2}

Anaplastic oligodendroglioma: features and $1 \mathrm{p} 19 \mathrm{q}$ status

\begin{tabular}{|lcc|}
\hline & 1p19q codeleted & 1p19q intact \\
\hline Histologic features [10••] & $\begin{array}{c}\text { Classic OD: monotonous, round cells; regular, round } \\
\text { nuclei; bland chromatin; perinuclear halo }\end{array}$ & $\begin{array}{c}\text { Some features of classic OD; astrocytic features, } \\
\text { GFAP positive }\end{array}$ \\
Imaging features $[17,18 \bullet, 23]$ & Indistinct border on T2 and FLAIR sequences \\
& Mixed signal intensity on T1 and T2 sequences \\
& Calcification present (best seen on CT) & Distinct borders on T2 and FLAIR sequences \\
& Possibly elevated thallium-201 and ${ }^{18 F}$ FDG uptake on & Homogeneous on T1 and T2 sequences \\
SPECT & Rare calcification \\
Localization $[50]$ & Frontal > parietal/occipital & - \\
Growth pattern $[18 \bullet]$ & Mixed or solid growth & Temporal, diencephalon, infratentorial
\end{tabular}

FLAIR—fluid-attenuated inversion recovery; GFAP—glial fibrillary acidic protein; OD—oligodendroglioma; SPECT—single-photon emission computed tomography. 


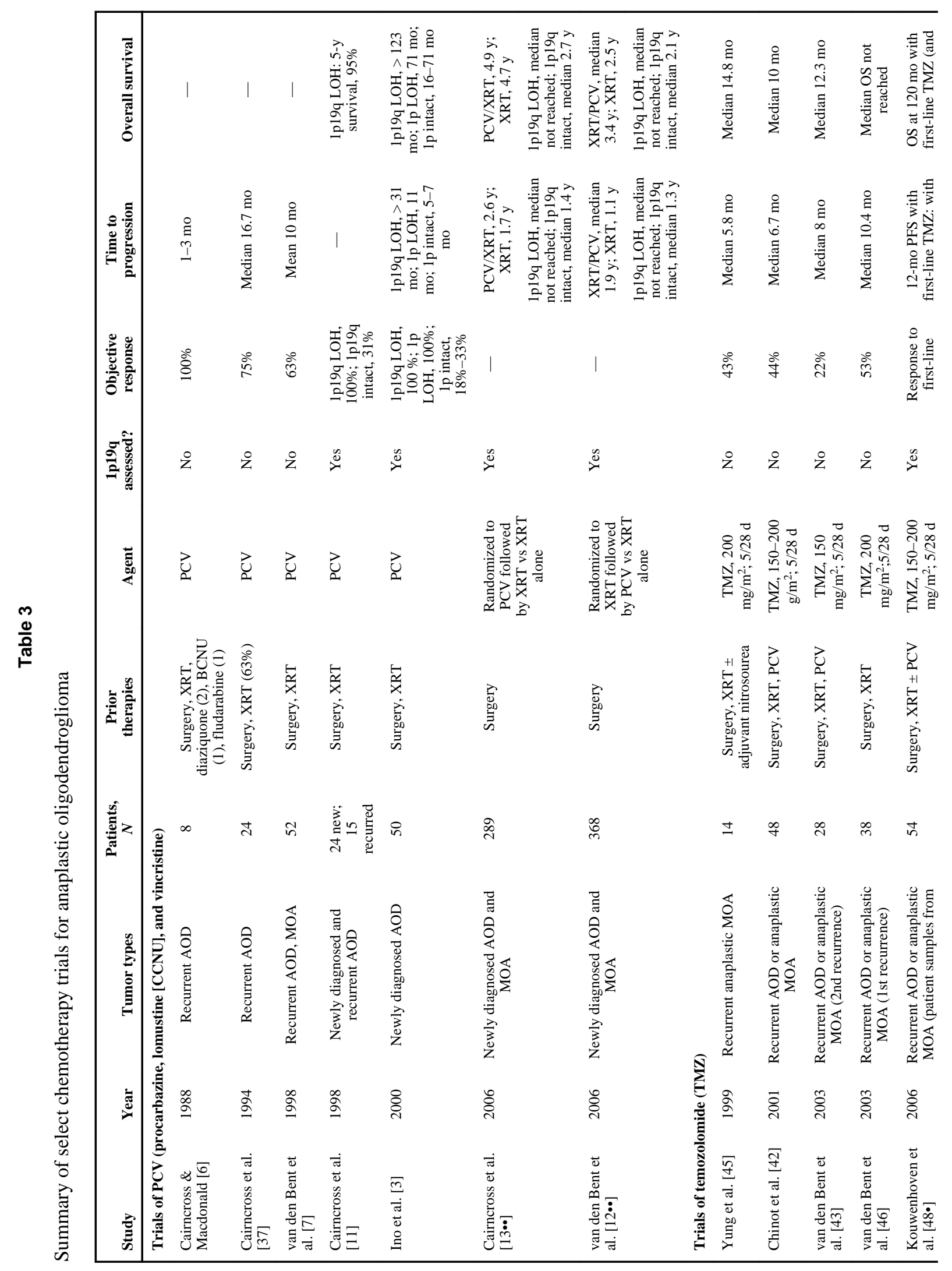




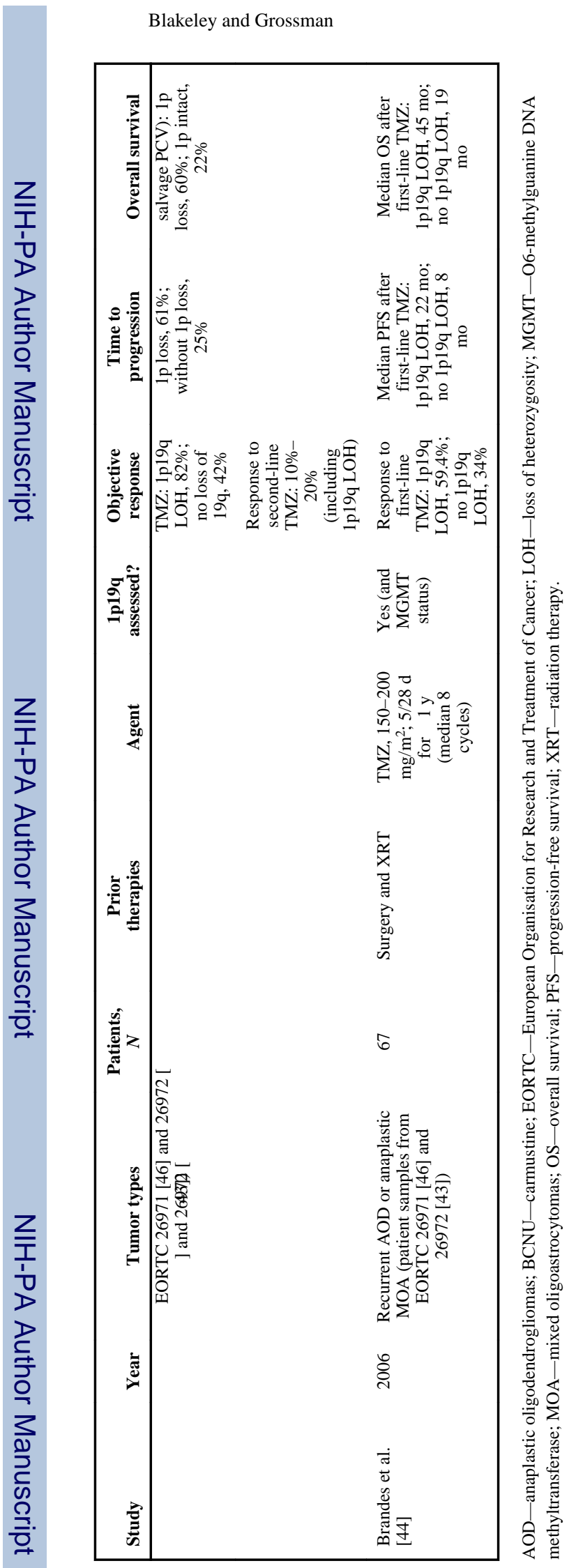

Curr Treat Options Neurol. Author manuscript; available in PMC 2014 April 22. 